\title{
In Silico Screening of Potential Spike Glycoprotein Inhibitors of SARS-CoV-2 with Drug Repurposing Strategy
}

\section{Tianzi Wei}

Department of Biology, Southern University of Science and Technology, Shenzhen, China; School of Biomedical Sciences, Li Ka Shing Faculty of Medicine, The University of Hong Kong, HKSAR, China

\section{Hao Wang}

Department of Obstetrics and Gynecology, Shenzhen University General Hospital, Shenzhen, Guangdong, China; Clinical Medical Academy, Shenzhen University, Guangdong, China

\section{Xueqing Wu}

Department of Obstetrics and Gynecology, Shenzhen University General Hospital, Shenzhen, Guangdong, China; Clinical Medical Academy, Shenzhen University, Guangdong, China

Yi Lu

School of Medicine, Southern University of Science and Technology, Shenzhen, China

\section{Shenghui Guan}

Department of Biology, Southern University of Science and Technology, Shenzhen, China

\section{Fengquan Dong}

Department of Cardiology, Shenzhen University General Hospital, Shenzhen, Guangdong, China; Clinical Medical Academy, Shenzhen University, Guangdong, China

\section{Chenle Dong}

Department of Obstetrics and Gynecology, The First Affiliated Hospital of Wenzhou Medical University, Wenzhou 325000, Zhejiang, China

\section{Guli Zhu}

Department of Obstetrics and Gynecology, Shenzhen University General Hospital, Shenzhen, Guangdong, China; Clinical Medical Academy, Shenzhen University, Guangdong, China

\section{Yuzhou Bao}

Department of Cardiology, Shenzhen University General Hospital, Shenzhen, Guangdong, China

\section{Jian Zhang ( $\square$ zhangjian@sustech.edu.cn )}

School of Medicine, Southern University of Science and Technology, Shenzhen, China

\section{Guanyu Wang ( $\nabla$ wanggy@sustech.edu.cn )}

Department of Biology, Southern University of Science and Technology, Shenzhen, China

Haiying Li ( $\nabla$ lihaiying111@hotmail.com )

Department of Cardiology, Shenzhen University General Hospital, Shenzhen, Guangdong, China; Clinical Medical Academy, Shenzhen University, Guangdong, China 


\section{Research Article}

Keywords: COVID19, SARS-CoV-2, Drug repurposing, Virtual screening, Spike glycoprotein, ACE2

Posted Date: April 6th, 2020

DOl: https://doi.org/10.21203/rs.3.rs-17720/v2

License: (c) (i) This work is licensed under a Creative Commons Attribution 4.0 International License. Read Full License

Version of Record: A version of this preprint was published at Chinese Journal of Integrative Medicine on August 1st, 2020. See the published version at https://doi.org/10.1007/s11655-020-3427-6. 


\section{Abstract}

COVID-19 has globally spread and has become a new pandemic, but there are still no effective drugs or vaccines to treat or prevent this disease. SARS-CoV-2 invades human cells through its spike proteins interacting with human ACE2 receptors, which may cause severe respiratory syndrome. One strategy to prevent the virus from entering cells is the interruption of the viral spike protein interacting with human ACE2. Facing such an urgent situation, drug repurposing is a promising strategy for rapid drug development. Here, we selected approximately 15000 molecular candidates, including FDA-approved drugs from DrugBank and natural compounds from TCMSP, to perform virtual screening for potential molecules that can target viral spike proteins, which may potentially interrupt the interaction between the human ACE2 receptor and viral spike protein. We found that digitoxin, a cardiac glycoside in DrugBank and bisindigotin, which is extracted from indigo naturalis and polygoni tinctorii foliu, in TCMSP had the highest docking scores. Note that indigo naturalis and the other herbs we found have been applied to prevent infectious diseases in traditional Chinese medicine. We also found that raltegravir, an HIV integrase inhibitor, has a relatively high binding affinity. All the docking results are presented in this article. Based on these docking results, further work will continue to identify potential molecules to prevent the spike protein from binding with the ACE2 receptor.

Authors Tianzi Wei and Hao Wang contributed equally to this work.

\section{Full Text}

\section{References}

1Zhou, P. et al. A pneumonia outbreak associated with a new coronavirus of probable bat origin. Nature, doi:10.1038/s41586-020-2012-7 (2020).

$2 \mathrm{Wu}, \mathrm{F}$. et al. A new coronavirus associated with human respiratory disease in China. Nature, doi:10.1038/s41586-020-2008-3 (2020).

3Wang, M. et al. Remdesivir and chloroquine effectively inhibit the recently emerged novel coronavirus (2019-nCoV) in vitro. Cell Res, doi:10.1038/s41422-020-0282-0 (2020).

4Belouzard, S., Chu, V. C. \& Whittaker, G. R. Activation of the SARS coronavirus spike protein via sequential proteolytic cleavage at two distinct sites. Proc Natl Acad Sci U S A 106, 5871-5876, doi:10.1073/pnas.0809524106 (2009).

5Zumla, A., Chan, J. F., Azhar, E. I., Hui, D. S. \& Yuen, K. Y. Coronaviruses - drug discovery and therapeutic options. Nat Rev Drug Discov 15, 327-347, doi:10.1038/nrd.2015.37 (2016).

6Wrapp, D. et al. Cryo-EM structure of the 2019-nCoV spike in the prefusion conformation. Science, doi:10.1126/science.abb2507 (2020). 
7Menger, L. et al. Trial watch: cardiac glycosides and cancer therapy. 2, e23082 (2013).

8Elbaz, H. A. et al. Digitoxin and its analogs as novel cancer therapeutics. 1, 4 (2012).

9Medicine, J. N. C. o. Dictionary of Traditional Chinese Drugs. (1986).

10Wei, X. Y. et al. Bisindigotin, a TCDD antagonist from the Chinese medicinal herb Isatis indigotica. 68, 427-429 (2005).

11Tang, X. et al. On the origin and continuing evolution of SARS-CoV-2. (2020).

12Wishart, D. S. et al. DrugBank 5.0: a major update to the DrugBank database for 2018. 46, D1074D1082 (2018).

13Ru, J. et al. TCMSP: a database of systems pharmacology for drug discovery from herbal medicines. 6 , 13 (2014).

14Trott, O. \& Olson, A. J. J. J. o. c. c. AutoDock Vina: improving the speed and accuracy of docking with a new scoring function, efficient optimization, and multithreading. 31, 455-461 (2010).

15DeLano, W. L. J. C. N. o. p. c. Pymol: An open-source molecular graphics tool. 40, 82-92 (2002).

16Systèmes, D. J. D. S. B. S. D., CA, USA. BIOVIA, Discovery Studio Modeling Environment. (2016).

\section{Introduction}

Since the middle of December 2019, a novel coronavirus disease (COVID-19) outbroke in Wuhan, the capital city of Hubei Province of China, and rapidly spread throughout the country with the population movement due to the Chinese New Year. Early studies utilized real-time PCR (RT-PCR) to confirm that the samples collected from one patient with pneumonia were positive for pan-betacoronavirus ${ }^{1}$. The viruses isolated from human cell lines were observed to contain typical crown-like shapes under a transmission electron microscope (TEM) with negative staining ${ }^{1}$.

Based on metagenomic sequencing, the whole-genome sequence of the virus was determined ${ }^{1,2}$. Bioinformatics analysis indicated that the novel virus causing severe pneumonia is a new type of betacoronavirus. The virus shares various typical genomic compositions with other betacoronavirus family members and has the highest sequence homology ( $96 \%$ sequence similarity) to SARS-like RaTG13 in bats ${ }^{1}$.

To date, COVID-19 cases have been reported in more than 67 countries. As of February 29, 2020, a cumulative total of 83,652 COVID-19 cases were reported worldwide. Although viral nucleic acid detection kits have been rapidly developed, effective drugs and vaccines are still being studied and are urgently needed. Facing such urgent situations, although necessary, developing new drugs or vaccines will require a very long time to complete, and drug repositioning is more rapid and possible to discover 
anti-SARS-CoV-2 drugs from approved drugs ${ }^{3}$. In addition, the natural compounds from traditional Chinese medicine (TCM) are potentially valuable pools for antivirus drug screening. Thus, rapid drug screening from these sources is an approach for anti-COVID-19 drugs.

Receptor recognition by coronavirus is the first and essential step for entering human cells. The homotrimeric spike glycoprotein (S protein), located on the envelope of SARS-CoV-2, is responsible for receptor recognition. The $\mathrm{S} 1$ subunit of the $\mathrm{S}$ protein, containing the receptor-binding domain (RBD), directly interacts with the receptor on the human cell membrane, while the $\mathrm{S} 2$ subunit facilitates virus-cell fusion and entry ${ }^{4}$. Angiotensin-converting enzyme 2 (ACE2) was shown to be the receptor mediating SARS-CoV-2 invasion in human cells ${ }^{1}$. Thus, interrupting the interaction between the $S$ protein and ACE2 is a strategy to inhibit virus entry ${ }^{5}$.

Recently, the three-dimensional structures of the homotrimeric spike glycoprotein (PDB ID: 6VSB) ${ }^{6}$ and the ACE2-B0AT1 complex were solved by cryo-EM. In addition, the complex structures of the receptorbinding domain (RBD) of the S protein bound to the human ACE2 were independently reported by three Chinese research teams. All related coordinates have been published by these researchers. Avoiding inaccurate protein structure modeling, these accurate coordinates of protein structures make it possible for rapid drug screening and vaccine design.

Here, we performed a structure-based virtual screening to search for molecules from the DrugBank and TCMSP databases to identify potential inhibitors targeting viral spike proteins. The selected binding pocket neighbors the interface between the viral spike protein and human receptor ACE2, which may further inhibit their interaction to prevent the virus from invading human cells. Following this computational work, more vigorous in vitro and in vivo experiments need to be performed. We hope this work will contribute to the discovery of anti-COVID-19 drugs.

\section{Results}

\subsection{Pocket identified on the Spike protein}

On February 19, 2020, Dr. Jianxun Qi's team published the crystal structure of the receptor-binding domain (RBD) of the spike protein complexed with the ACE2 receptor on the National Microbiology Data Center (No. NMDCS0000001; PDB ID: 6LZG). According to this crystal structure, Discovery Studio 2016 was employed to detect the binding pocket on the spike protein. In this work, the second-ranked pocket was selected as its position near the protein-protein interface and may induce a conformational change to intervene in the interaction (see Figure 1). The coordination of the pocket is as follows: center $x=-$ 56.387 , center $y=52.408$, center $z=21.937$; size $x=25$, size $y=25$, size $z=25$.

\subsection{Data source}


A total of 2,191 compounds from DrugBank and 13,026 compounds from TCMSP were prepared as candidates. AutoDock Vina was employed to perform in silico high-throughput screening. The compounds in DrugBank are all FDA-approved compounds. The compounds provided by TCMSP are natural compounds. These compounds are traditional Chinese medicine (TCM). All molecular structure files were optimized by the force field MMFF94.

\subsection{Docking results of molecules from the DrugBank dataset.}

For the DrugBank dataset, Table 1 lists the top 10 compounds with the highest binding energy. The specific screening results for each compound are given in the supplementary material.

The compound digitoxin was identified as having the strongest binding energy, $-8.7 \mathrm{kcal} / \mathrm{mol}$, at the binding site. Digitoxin is a cardiac glycoside. It has a known target, the sodium/potassium-transporting ATPase subunit. Digitoxin can be used to treat patients with heart failure. Scientists have found that digitoxin is also a potential anticancer drug ${ }^{7,8}$. However, digitoxin can cause toxicity to the human body, including causing nausea, anorexia, confusion and so on. Figures 2 and 3 show the interaction of digitoxin with the spike protein.

Figures 4 and 5 represent the interactions among the S protein and ACE2 and the binding site of digitoxin on the complex.

\subsection{Docking results of the TCMSP dataset.}

The top 10 binding results of the TCMSP dataset are shown in Table 2. The complete binding information of TCMSP can be found in the supplementary material.

The compound with the highest binding energy in the TCMSP dataset with the S protein is bisindigotin, which can be isolated from Isatis indigotica and Polygoni Tinctorii Foliu. Both herbs have heat-clearing and detoxifying effects in the theories of traditional Chinese medicine. Lsatis indigotica is a folk medicine used to treat viral disease and inflammatory disease ${ }^{9}$. In addition, Wei et al. reported that bisindigotin can act as an antagonist to relieve the hepatoxicity caused by 2,3,7,8-tetrachlorodibenzo- $p$-dioxin, which is a carcinogen ${ }^{10}$. Figures 6 and 7 show the interaction between bisindigotin and RBD of the S protein.

Figures 8 and 9 demonstrate the S protein trimer structure complexed with the ACE2 protein and the binding site of bisindigotin.

\section{Discussion}

The world is now confronted with a public health crisis caused by SARS-CoV-2. State-of-the-art techniques need to be applied to solve this pandemic. Normally, de novo drug development is a time- 
consuming project. Therefore, drug repurposing theories may be helpful to find the drug preventing coronavirus as soon as possible.

Here, we first chose the spike protein rather than the ACE2 receptor for a binding pocket search because ACE2 is expressed in various types of human cells, and targeting ACE2 might cause more side effects. We observed that the cavity neighboring the interface between the viral spike protein and the human ACE2 receptor is the second ranked pocket. Targeting this position may contribute to interrupting the interaction between the S protein and the ACE2 receptor. Due to its high ranking and position advantages, we used this cavity for the next docking progress. Future work might focus on identifying a suitable cavity within the interface formed by the viral spike protein and human receptor ACE2.

In this work, two databases, DrugBank, containing FDA-approved drugs, and TCMSP, with TCM bioactive molecules, were used to explore potential candidates to prevent SARS-CoV-2 from invading the human body. The docking results show that the molecules having the strongest binding energy from DrugBank and TCMSP are digitoxin and bisindigotin, respectively. Bisindigotin is a bioactive TCM from /satis indigotica. This plant has been used as an herb in China to treat viral disease. It may have great potential to serve as a promising drug to treat COVID-19. More vigorous work remains to be done to test these molecules' effects.

Additionally, in the top 10 list of DrugBank docking results, it is worth noting that an HIV antiretroviral drug, raltegravir, was identified. This is a new class of HIV drugs that can inhibit HIV integrase. On the other hand, in the top 10 list of TCMSP, in addition to bisindigotin, we observed that many herbs contain molecules that have effects on clearing heat, and these include indigo naturalis, isatidis radix, isatidis folium, stemonae radix, wikstroemiae indicae rasix, ginkgo semen, platycladi cacumen, forsythiae fructus, ranunculi ternati radix, canarii fructus, selaginella doederleinii hieron, and ginkgo folium. According to TCM theories, these herbs can be used to relieve symptoms of pneumonia. Importantly, according to the national clinical guidelines, certain abovementioned herbs have been regarded as components of TCM for treating COVID-19 patients who have mild-to-moderate disease.

SARS-CoV-2 is not stable as it is an RNA virus. Scientists have found that it may have mutations generating another subtype ${ }^{11}$. However, the critical proteins in SARS-CoV-2 are still conserved. Currently, the possible mutations that occur will have a significant impact on our work. In future research, we will investigate more molecules that have a lower binding energy and rank than those of digitoxin and bisindigotin. Moreover, in vitro tests will be carried out to verify the binding between the $\mathrm{S}$ protein and the molecules identified by computational methods.

\section{Methods And Materials}

\subsection{DrugBank}

DrugBank is a drug data resource that contains comprehensive drug information, including approved and experimental small-molecule, biologics, and nutraceutical data ${ }^{12}$. The latest version of DrugBank was 
released on January 13,2020 . It currently has 2,628 approved small drugs. Before performing virtual screening, approved drugs with molecular weights larger than $500 \mathrm{kDa}$, including polypeptides, were deleted; thus, only 2,191 FDA-approved drugs were screened.

\subsection{TCMSP}

TCMSP was developed by Northwest University in China, which is a platform providing information connecting bioactive molecules in TCM, targets, and diseases in a network. In addition, this platform also provides related pharmacokinetic properties ${ }^{13}$. Based on this platform, 13,026 small molecules from TCM herbs were screened.

\subsection{Software used}

AutoDock Vina 1.1.2 $2^{14}$ was used in this work to perform visual screening. PyMOL 2.3.3 ${ }^{15}$ and Discovery Studio $2016^{16}$ were used to exhibit the ligand-receptor interactions.

\section{Declarations}

\section{Acknowledgment}

Great thanks to Dr. Jianxun Qi for working to solve the crystal structure of the RBD-ACE2 complex. Dr. Jianxun Qi gave us permission to use this crystal structure in this study. We appreciate the support from Southern University of Science and Technology and Shenzhen University General Hospital.

\section{Conflict of interest}

The authors disclose no conflicts.

\section{Ethics statement}

This work was approved by the Institutional Review Board (IRB) of Southern University of Science and Technology.

\section{References}

1Zhou, P. et al. A pneumonia outbreak associated with a new coronavirus of probable bat origin. Nature, doi:10.1038/s41586-020-2012-7 (2020).

$2 \mathrm{Wu}, \mathrm{F}$. et al. A new coronavirus associated with human respiratory disease in China. Nature, doi:10.1038/s41586-020-2008-3 (2020). 
3Wang, M. et al. Remdesivir and chloroquine effectively inhibit the recently emerged novel coronavirus (2019-nCoV) in vitro. Cell Res, doi:10.1038/s41422-020-0282-0 (2020).

4Belouzard, S., Chu, V. C. \& Whittaker, G. R. Activation of the SARS coronavirus spike protein via sequential proteolytic cleavage at two distinct sites. Proc Natl Acad Sci U S A 106, 5871-5876, doi:10.1073/pnas.0809524106 (2009).

5Zumla, A., Chan, J. F., Azhar, E. I., Hui, D. S. \& Yuen, K. Y. Coronaviruses - drug discovery and therapeutic options. Nat Rev Drug Discov 15, 327-347, doi:10.1038/nrd.2015.37 (2016).

6Wrapp, D. et al. Cryo-EM structure of the 2019-nCoV spike in the prefusion conformation. Science, doi:10.1126/science.abb2507 (2020).

7Menger, L. et al. Trial watch: cardiac glycosides and cancer therapy. 2, e23082 (2013).

8Elbaz, H. A. et al. Digitoxin and its analogs as novel cancer therapeutics. 1, 4 (2012).

9Medicine, J. N. C. o. Dictionary of Traditional Chinese Drugs. (1986).

10Wei, X. Y. et al. Bisindigotin, a TCDD antagonist from the Chinese medicinal herb Isatis indigotica. 68 , 427-429 (2005).

11Tang, X. et al. On the origin and continuing evolution of SARS-CoV-2. (2020).

12Wishart, D. S. et al. DrugBank 5.0: a major update to the DrugBank database for 2018. 46, D1074D1082 (2018).

13Ru, J. et al. TCMSP: a database of systems pharmacology for drug discovery from herbal medicines. 6 , 13 (2014).

14Trott, O. \& Olson, A. J. J. J. o. c. c. AutoDock Vina: improving the speed and accuracy of docking with a new scoring function, efficient optimization, and multithreading. 31, 455-461 (2010).

15DeLano, W. L. J. C. N. o. p. c. Pymol: An open-source molecular graphics tool. 40, 82-92 (2002).

16Systèmes, D. J. D. S. B. S. D., CA, USA. BIOVIA, Discovery Studio Modeling Environment. (2016).

\section{Tables}

Due to technical limitations, Tables 1-2 are provided in the Supplementary Files section.

\section{Figures}




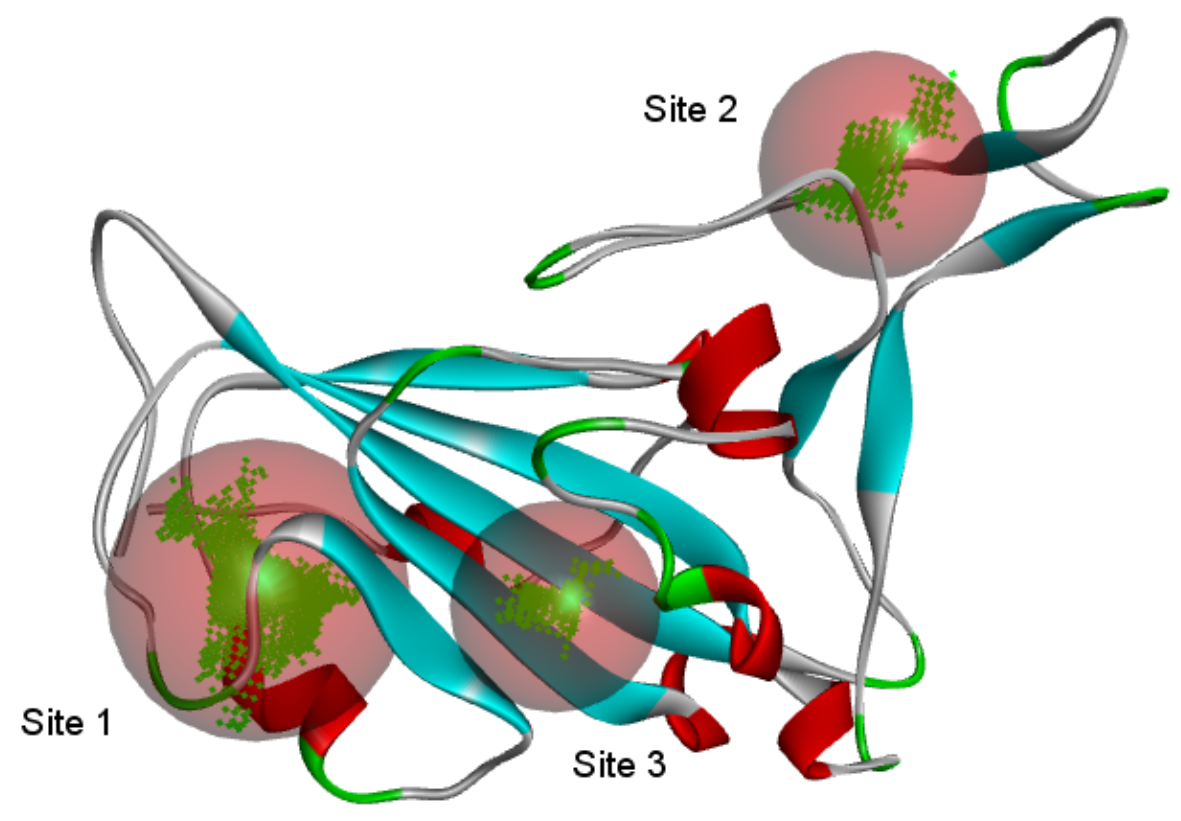

\section{Figure 1}

Top 3 pockets predicted by Discovery Studio 2016. The pockets are labeled according to their rank.

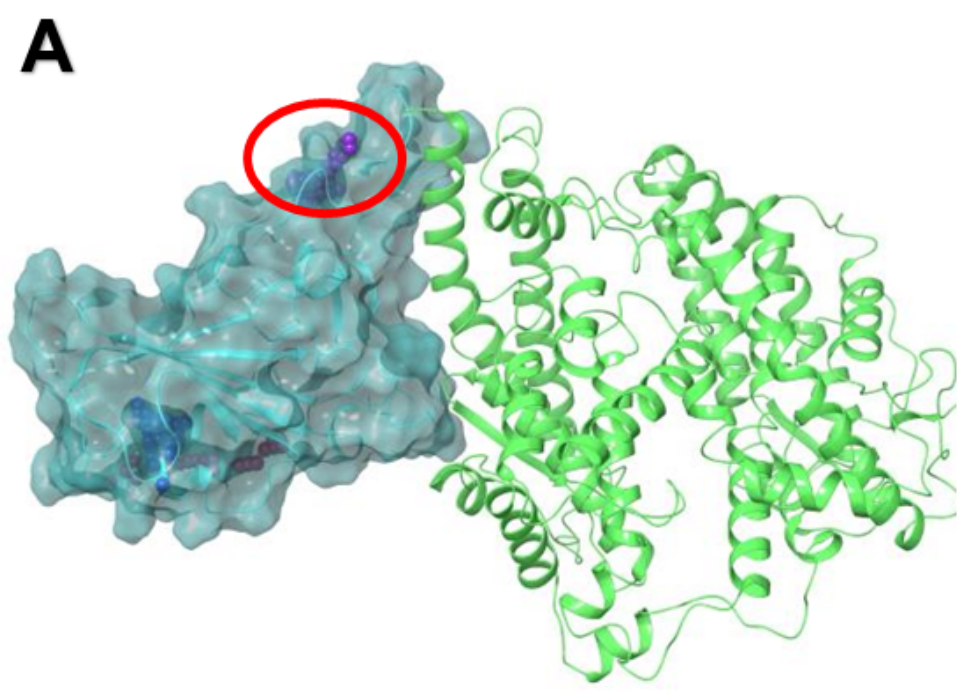

B

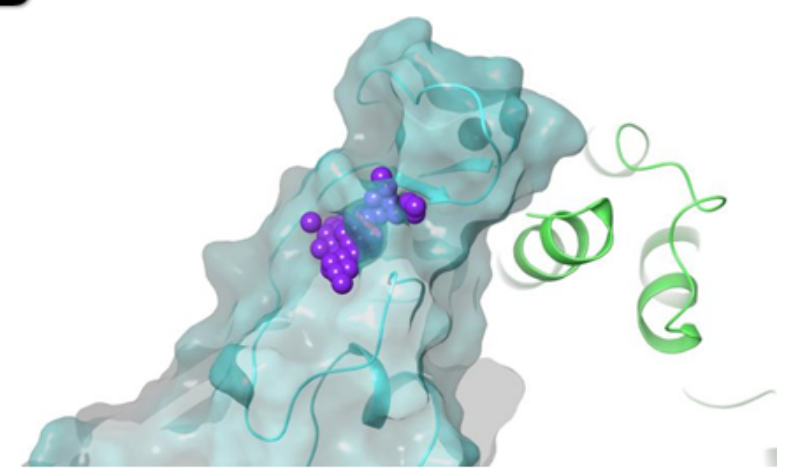

Figure 2

(A) The position circled in red is the pocket selected. The protein colored green is ACE2. (B) The position consisting of purple balls is the pocket selected. 


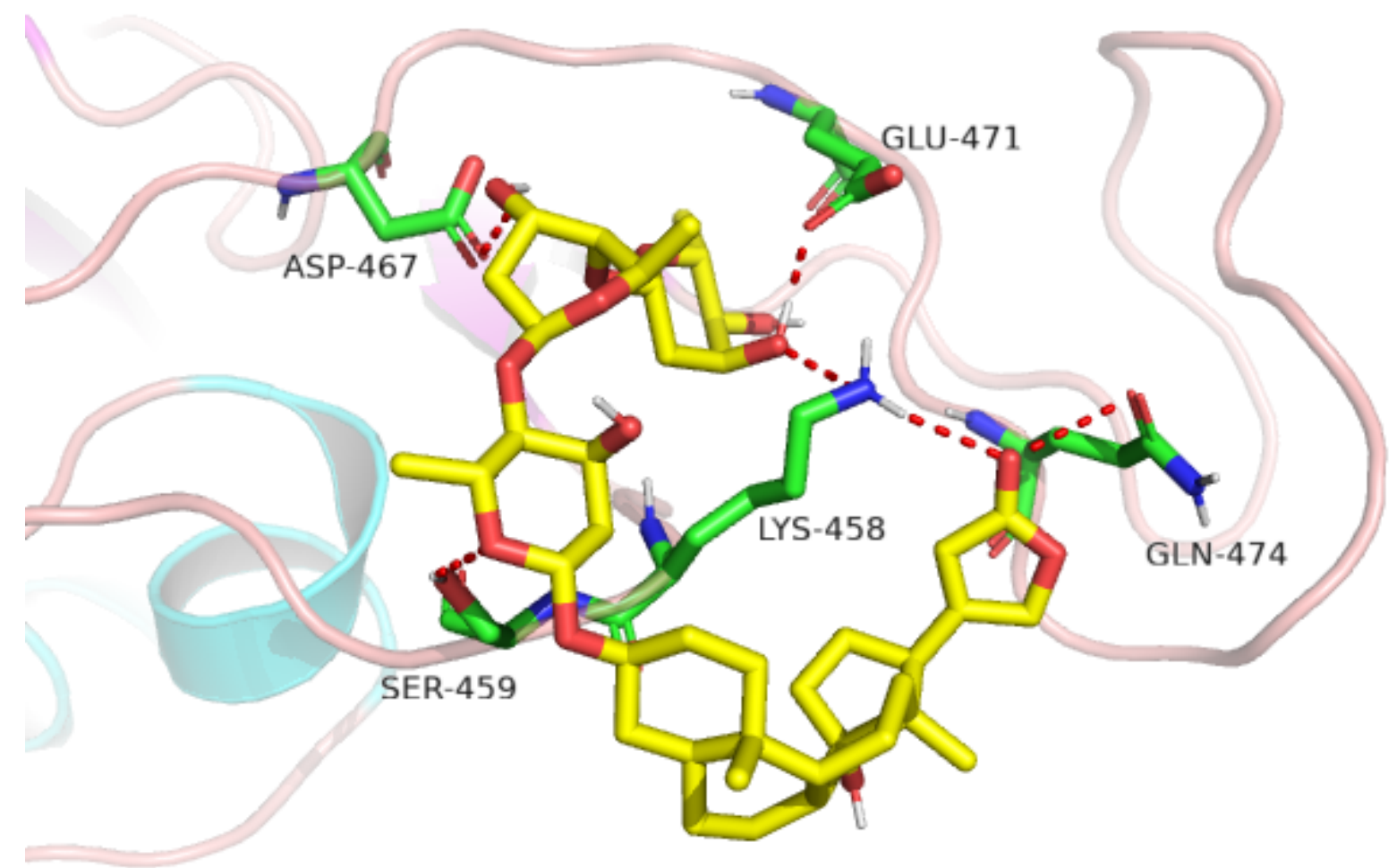

Figure 3

3D interactions between digitoxin (yellow) and RBD of the S protein (green sticks, pink loops, and blue helix). The red dotted line represents polar contacts. 


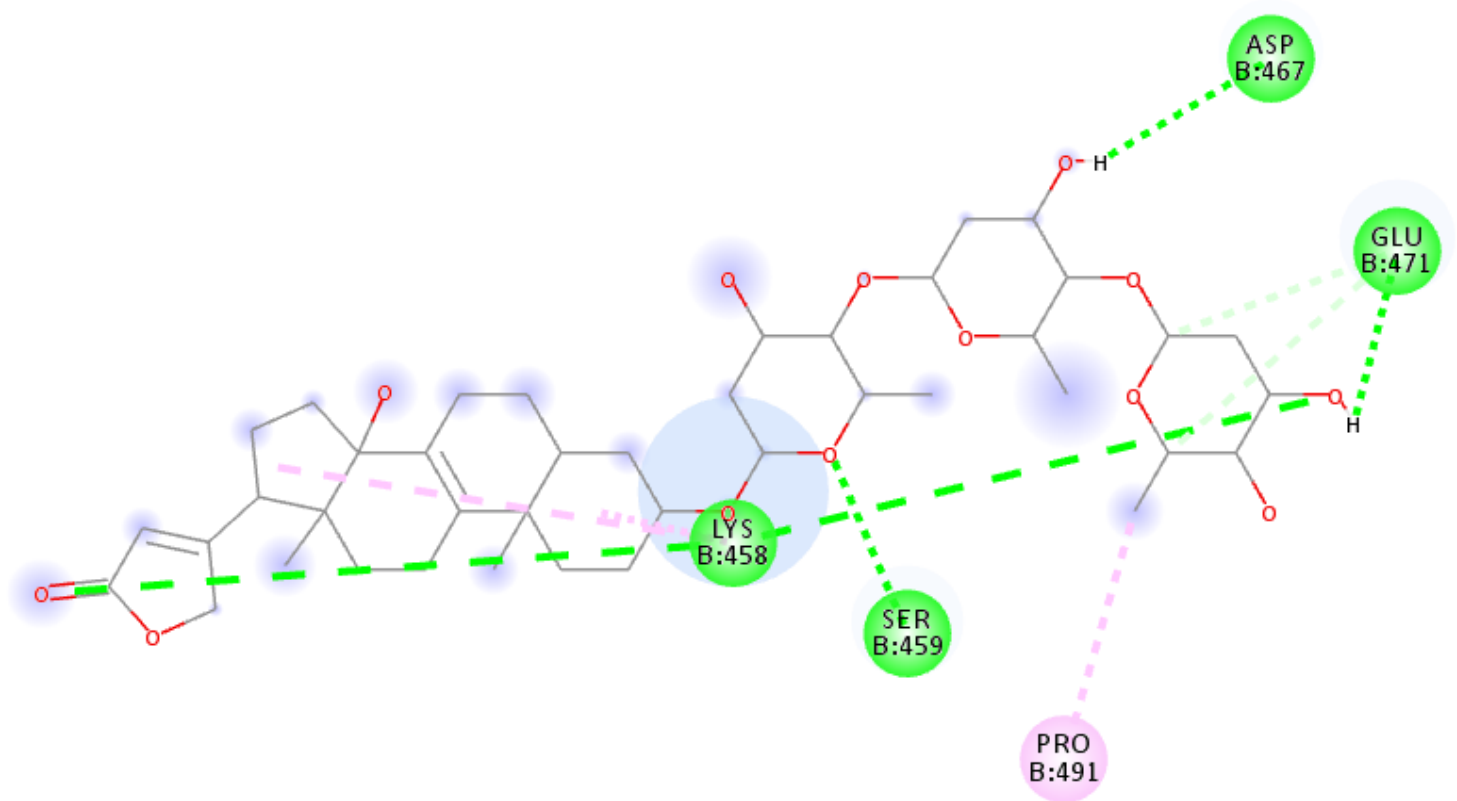

Interactions

Conventional Hydrogen Bond

Carbon Hydrogen Bond

\section{Figure 4}

2D interactions between digitoxin and RBD of the S protein.

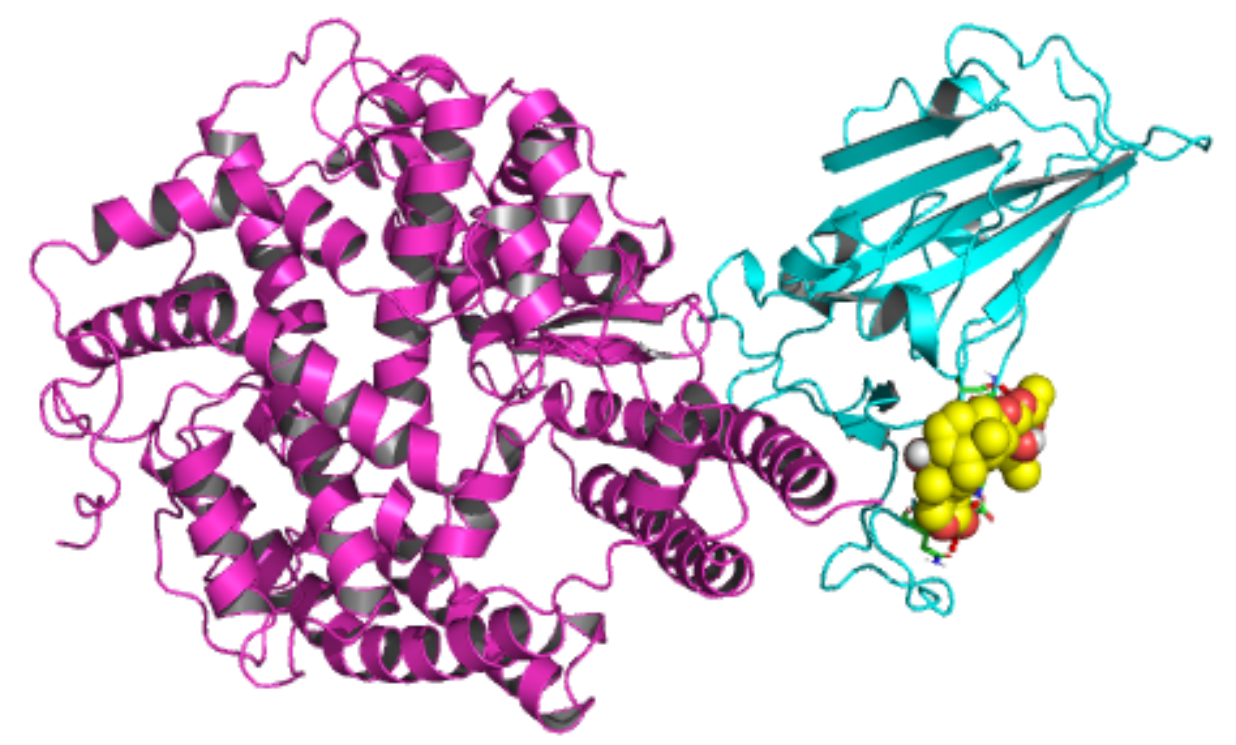

Figure 5 
Digitoxin on the interface between RBD and ACE2. The protein colored magenta is ACE2; the blue represents the $\mathrm{S} 1$ subunit of the $S$ protein. The balls colored yellow, red and white are digitoxin.

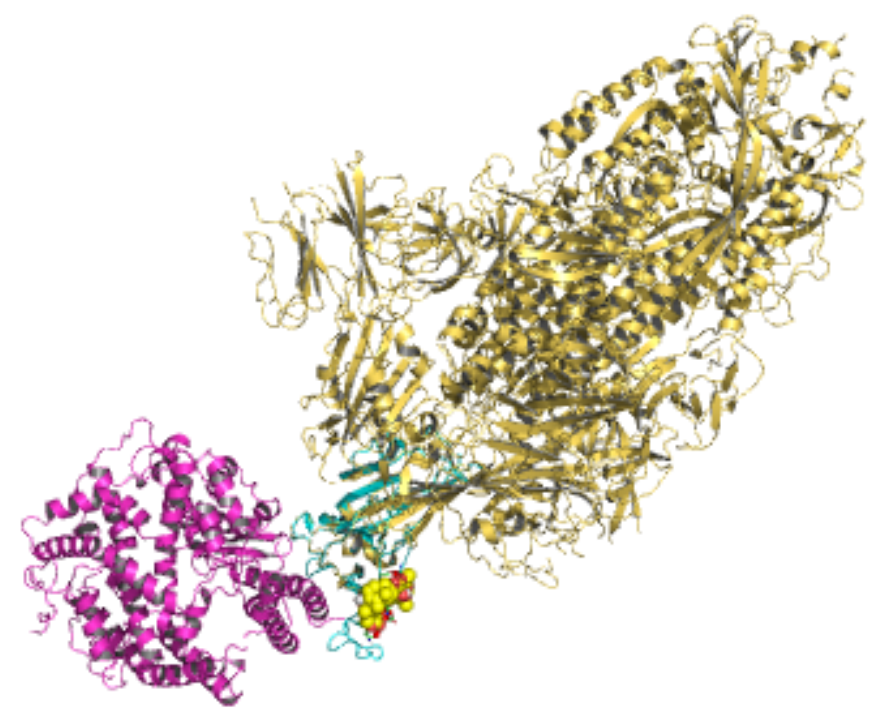

Figure 6

Digitoxin on the interface between the S protein and ACE2. The protein colored magenta is ACE2; the blue represents the $\mathrm{S} 1$ subunit of the S protein. The balls colored yellow, red and white are digitoxin. The trimer $S$ protein (golden) is superposed on the RBD-ACE2 complex. 


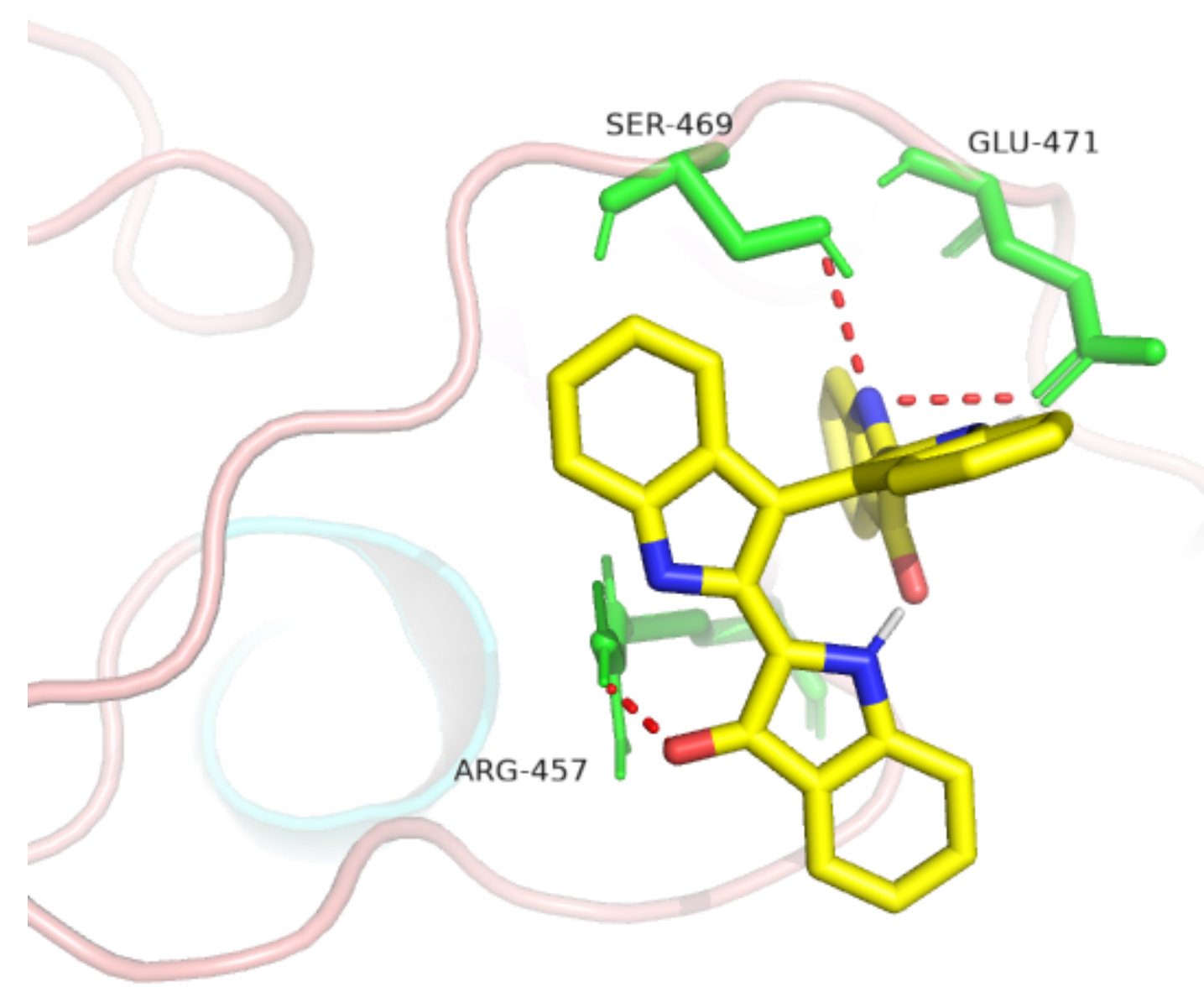

Figure 7

3D interactions between bisindigotin (yellow) and RBD of the S protein (green sticks, pink loops, and blue helix). The red dotted line represents polar contacts. 


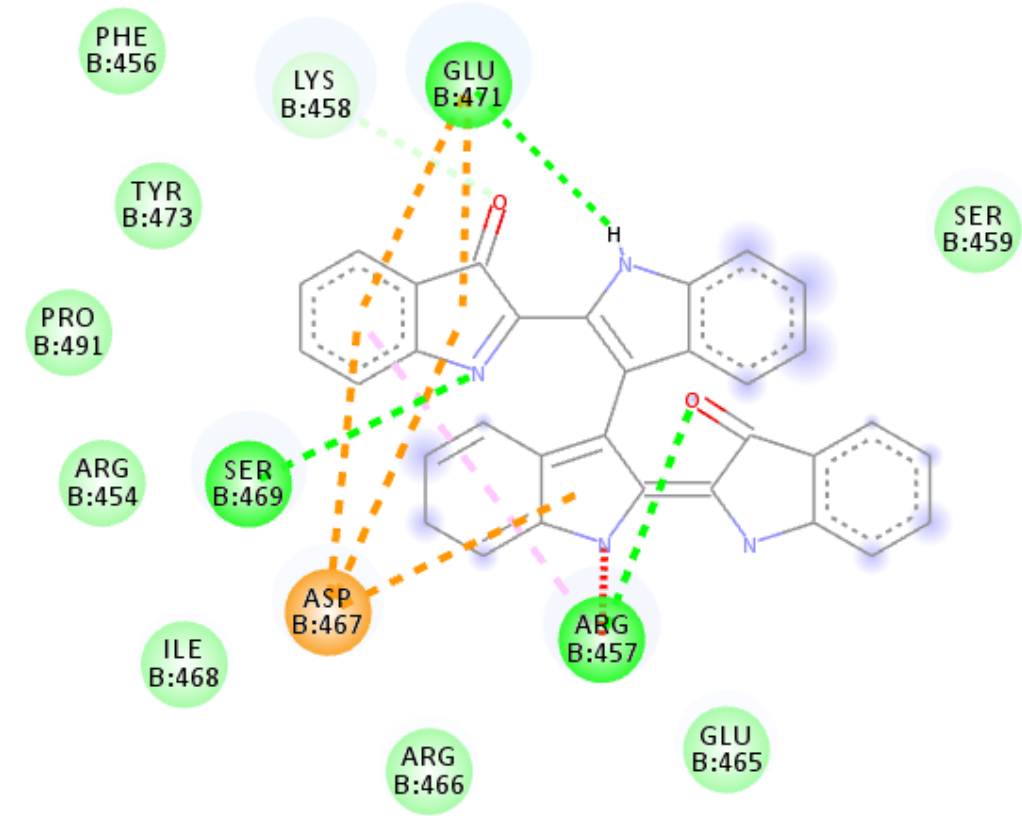

Interactions

van der "llasls

Conventional Hydrogen Bond

Carbon Hydrogen Bond

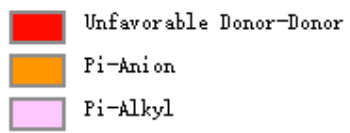

Figure 8

$2 \mathrm{D}$ interactions between bisindigotin and RBD of the $S$ protein.

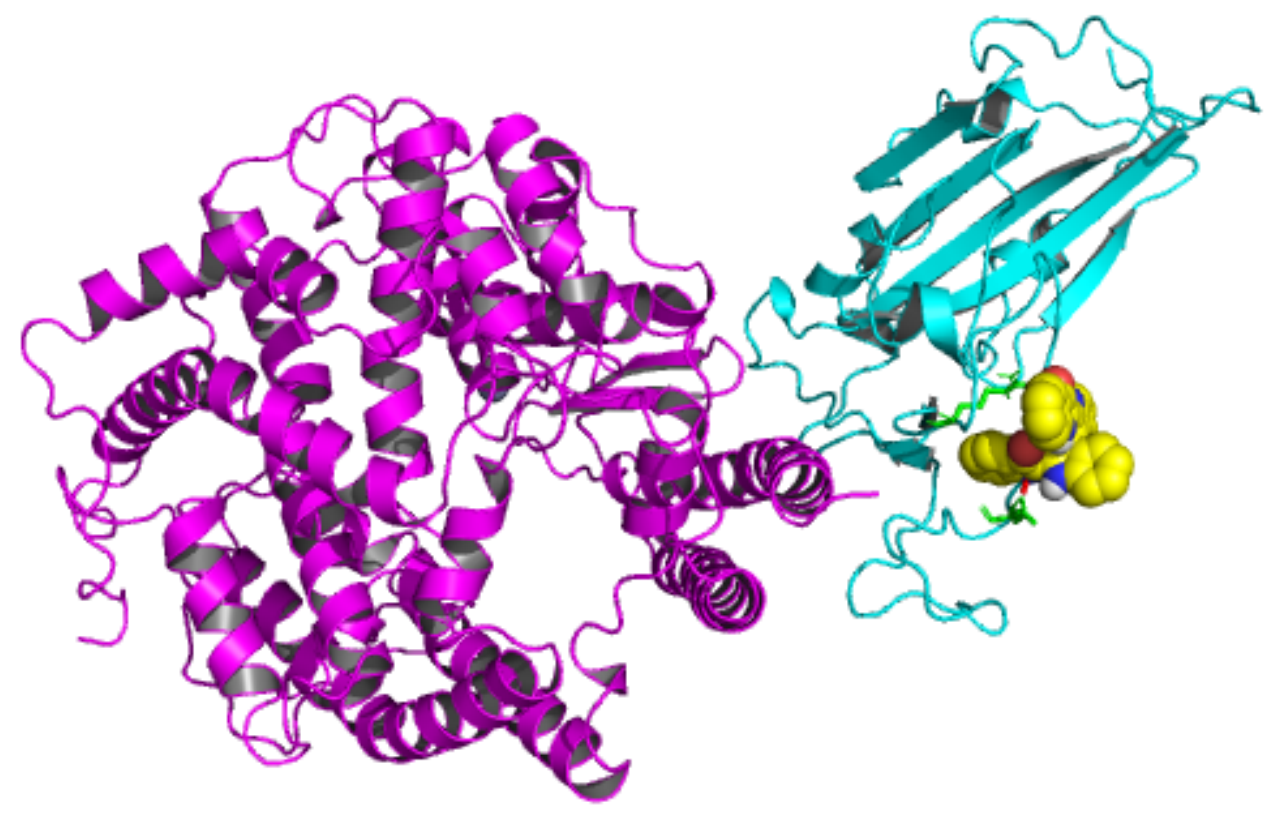

Figure 9 
Bisindigotin on the interface between the RBD of The S protein and ACE2. The blue part is the S1 subunit of the S protein. The balls colored yellow, red and white are bisindigotin; the protein colored magenta is ACE2.

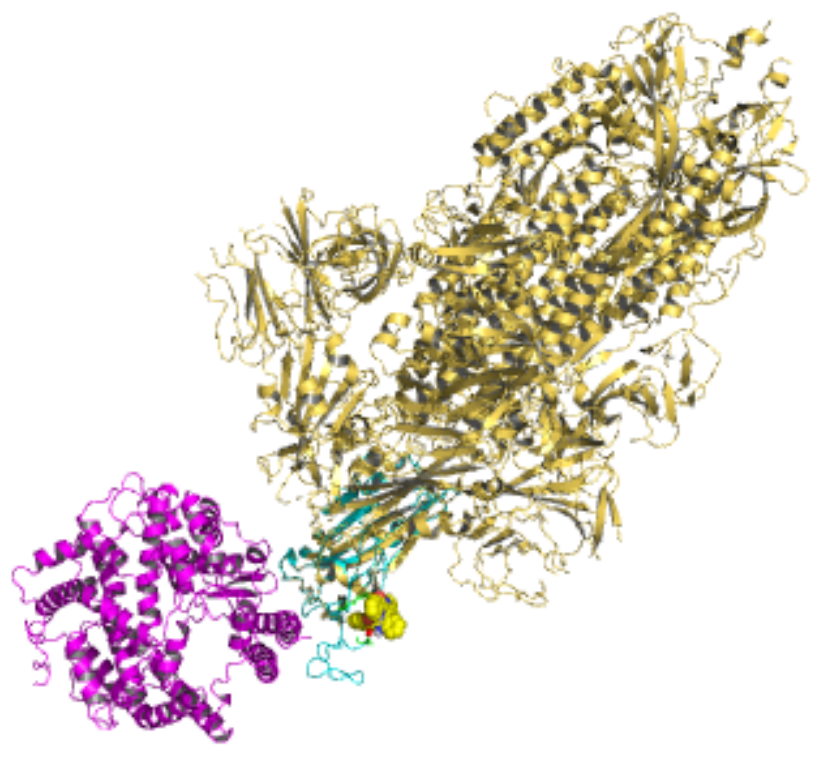

Figure 10

Bisindigotin on the interface between the S protein and ACE2. The protein colored magenta is ACE2; the blue represents the $\mathrm{S} 1$ subunit of the $\mathrm{S}$ protein. The balls colored yellow, red and white are digitoxin. The trimer S protein (golden) is superposed on the RBD-ACE2 complex.

\section{Supplementary Files}

This is a list of supplementary files associated with this preprint. Click to download.

- graphicabstract.png

- supplementalmaterial.zip

- table12.docx 